\title{
BreathSens: A Continuous On-Bed Respiratory Monitoring System With Torso Localization Using an Unobtrusive Pressure Sensing Array
}

\author{
Jason J. Liu, Student Member, IEEE, Ming-Chun Huang, Student Member, IEEE, Wenyao Xu, Member, IEEE, \\ Xiaoyi Zhang, Student Member, IEEE, Luke Stevens, Nabil Alshurafa, Student Member, IEEE, \\ and Majid Sarrafzadeh, Fellow, IEEE
}

\begin{abstract}
The ability to continuously monitor respiration rates of patients in homecare or in clinics is an important goal. Past research showed that monitoring patient breathing can lower the associated mortality rates for long-term bedridden patients. Nowadays, in-bed sensors consisting of pressure sensitive arrays are unobtrusive and are suitable for deployment in a wide range of settings. Such systems aim to extract respiratory signals from timeseries pressure sequences. However, variance of movements, such as unpredictable extremities activities, affect the quality of the extracted respiratory signals. BreathSens, a high-density pressure sensing system made of e-Textile, profiles the underbody pressure distribution and localizes torso area based on the high-resolution pressure images. With a robust bodyparts localization algorithm, respiratory signals extracted from the localized torso area are insensitive to arbitrary extremities movements. In a study of 12 subjects, BreathSens demonstrated its respiratory monitoring capability with variations of sleep postures, locations, and commonly tilted clinical bed conditions.
\end{abstract}

Index Terms-Pictorial structure, pressure sensor, respiration monitoring, signal extraction.

\section{INTRODUCTION}

$\mathbf{U}$ RGENT medical care is required when patients have abnormal respiration such as tachypnea (high respiration), bradypnea (low respiration), or apnea (no respiration). Peberdy et al. reported that $44 \%$ of over 14000 cardiac arrests in acute care hospitals were attributed to respiratory problems [1]. Furthermore, the 2011 HealthGrades study showed that $20 \%$ of

Manuscript received May 16, 2014; revised July 21, 2014; accepted July 23, 2014. Date of publication July 31, 2014; date of current version September 1 , 2015.

J. J. Liu, N. Alshurafa, and M. Sarrafzadeh are with the Wireless Health Institute, Department of Computer Science, University of California, Los Angeles, CA90095 USA (e-mail: jasonliu@cs.ucla.edu; nabil@cs.ucla.edu; majid@cs. ucla.edu).

M.-C. Huang is with the Electrical Engineering and Computer Science, Case Western Reserve University, Cleveland, OH 44106 USA (e-mail: ming-chun. huang@case.edu).

W. Xu is with the Department of Computer Science and Engineering, State University of New York at Buffalo, New York, NY 14260 USA (e-mail: wenyaoxu@buffalo.edu).

$\mathrm{X}$. Zhang is with the Computer Science and Engineering, University of Washington, Seattle, WA 98195 USA (e-mail: xiaoyiz@cs.washington.edu).

L. Stevens is with Medisens Wireless, Inc., Santa Clara, CA 95051 USA (e-mail: luke@medisens.com).

Color versions of one or more of the figures in this paper are available online at http://ieeexplore.ieee.org.

Digital Object Identifier 10.1109/JBHI.2014.2344679 postoperative respiratory failures in 5000 hospitals resulted in death [2].

Hodgetts et al. [3] and Fieselmann et al. [4] observed that a large proportion of cardiac arrest patients had high-respiration rate. In addition, the variation in respiratory rate is another important marker for cardiac arrest or admission to the intensive care unit [5].

Despite such information, respiration rate monitoring has not received the same level of attention compared with monitoring of the other vital signs such as blood pressure and heart rate [6]. McBride et al. showed the lack of consideration toward respiratory rate reporting that only $30 \%$ of patients have their respiratory rate recorded daily [7]. Reasons include the lack of reliable and unobtrusive respiration rate measurement systems [5]. Hospitals pay less attention to respiratory rates due to the fact that current monitoring systems require direct contact with the skin [8]. Other methods such as video analysis to infer breath rate are noncontact; however, there are issues with privacy and low-light level at night. Thus, in many hospitals, medical personnel manually measure breathing rates.

This paper introduces BreathSens, a system that unobtrusively monitors on-bed respiration. By targeting the torso region of the body, this system converts the pressure distribution to a respiratory signal. We summarize the innovations as

1) design of an indirect contact respiration rate monitoring system that has better usability and comfort due to body localization;

2) a framework that applies a torso localization algorithm to enable measurement of respiration rate;

3) results of experimental studies in both controlled and tilted bed environments.

Furthermore, we also show a method of visualization of the breathing patterns during regular sleep periods. This study focuses on respiration monitoring over continuous periods with the purpose of measuring average respiration rates over extended time periods or during sleep.

The remainder of this paper is organized as follows. Section II describes current related work in measurement of respiration rate. Section III details briefly the pressure sensitive bedsheet used for respiration monitoring. The algorithmic method is described in Section IV, and the experimental setup and results are given in Section V. Finally, a discussion and future work are given in Section VI. 


\section{RELATED WORK}

This section describes the current state of the art in measuring respiration rate. There are three main categories of respiratory rate monitoring: on-body, indirect contact, and noncontact.

On-body sensors are either directly attached on the skin or wearable through straps. The main concern with on-body sensors is their obtrusive nature and setup issues. Hospitals use pulse oximeters, such as the Nellcor Respiratory Monitor [9], to estimate respiratory rate from oximetry measurements on the patient's finger. However, the results are often inaccurate when patients have abnormal levels of oxygen saturation or low pulses [10]. Respiratory inductance plesthysmography is a dual belted sensor worn around the chest and abdomen. As patient's body expands and contracts during breathing, this sensor reflects changes in the inductance of its coils. Now, many sleep centers use this sensor solution, which is recommended by the American Academy of Sleep Medicine [11].

The second category of respiratory rate monitoring is indirect contact. Typically, this method involves sensors embedded in the mattress, sheet, or pillow, which overcomes the discomfort issues inherent with on-body sensors. An air mattress sensor system [12] allows measurement of the respiration and heart beat movements without use of any on-body sensor. However, the shape, type, or thickness of the mattress may introduce noise to the sensed data. Another type of indirect contact sensor is capacitance sensor, which measures the changes in electrical permittivity above the sensor caused by air in the lungs [13]. Again, it required the subject to be localized directly above the sensor. Using hetero-core fiber optic pressure sensors, Nishyama et al. [14] relaxed the restriction of sleep pose a little further by having a wider sensitivity to small pressure changes and could account for large changes.

The third category of respiratory rate monitoring is noncontact. For instance, using video or other electromagnetic radiation sensing [15]. Current developments in video analyses can measure respiratory rate and heart rate by analyzing the small changes in color of subjects' faces [16]. The Philips Vital Signs Camera [17] application on iOS devices performs this contactless measurement of breathing rate. However, using video cameras leads to a concern about patient privacy in hospital and lighting during the night can be a significant problem. The use of infrared sensors can diminish some of these issues. Murthy et al. used an infrared imaging system to detect the temperature change of exhaled air out of patients' mouths [18].

In summary, noncontact-based respiratory rate monitoring has the advantage of noninvasiveness, however, still needs to be localized, while contact sensors are obtrusive and need manual setup. On the other hand, our system, a high-density pressure sensitive bed sheet, is noninvasive, comfortable, and can measure respiration at any location on the mattress. It is able to provide sufficient accuracy to be used as a screening tool for changes in respiration.

\section{Pressure-Sensitive BedsheEt System}

The designed prototype bed sheet is a $2.5 \mathrm{~m} \times 1.25 \mathrm{~m}$ system that contains $64 \times 128$ pressure sensors (see Fig. 1). Sixty-four

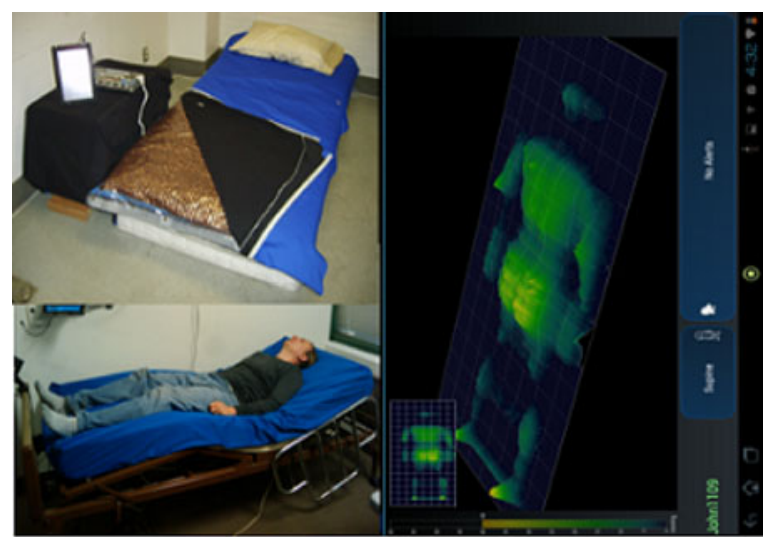

Fig. 1. High-density pressure sensor array captures a full pressure distribution. The pressure is represented as pixel intensities.

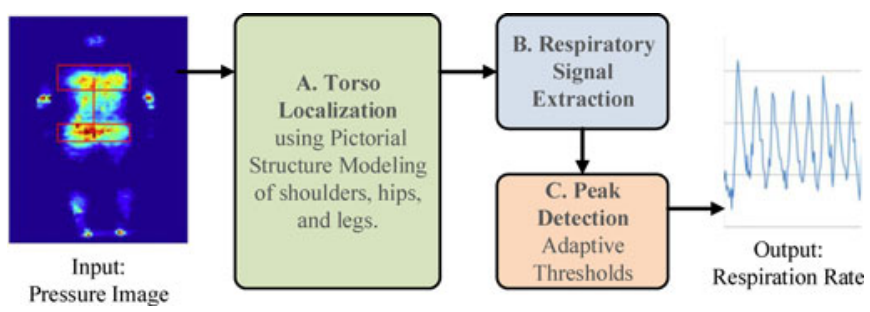

Fig. 2. Respiration monitoring flow consists of three components. (a) Torso localization. (b) Respiratory signal extraction. (c) Peak detection.

column conductive lines and 128 row conductive lines generate 8192 intersections. A sheet of e-Textile fabric, which is regular fabric coated with piezoelectric polymer is located in between the row and column layers. Therefore, each joint intersection forms a pressure sensor within the three-layer sandwich structure (the resistance of the e-Textile changes when pressure is applied [19], [20]). Because of the fabric, the feel of the sensing system is just like regular fabric.

A sampling unit is connected to all conductive lines and performs matrix scanning to measure pressure map sequences. Retrieved pressure map signals of the 8192 sensors are quantified to values ranging from 0 to 255 [21], [22]. Sampling rate is adjustable up to $10 \mathrm{~Hz}$. For this respiratory rate measurement, a sampling rate of $1.5 \mathrm{~Hz}$ was used. This allows the system to achieve a maximum breathing detection rate up to 45 breaths per minute according to the Nyquist rule.

\section{BREATH MONITORING AlgORITHM}

The method for monitoring respiration rate is composed of three parts: torso localization, respiratory signal extraction, and peak detection (see Fig. 2). Locating the torso area in the timeindexed pressure images reduces interference caused by extremities movement. The torso localization algorithm is based on a pictorial model and cost minimization method. Mismatch and deformation costs combine as the overall cost to most accurately fit a pictorial model into a pressure image. The torso localization algorithm aims to find the best fit among all possible body model configurations in the pressure image and so the 
high-density bedsheet enables accurate body localization. Once the torso area is localized, the pressure values in the area of chest and stomach are used to extract respiratory signals using a vertical weighting calculation. A peak detection algorithm with adaptive thresholding is applied to mark breathing events and intervals. Fig. 2 shows the overview of the algorithm.

\section{A. Torso Localization}

The torso localization method is based on the pictorial structures model proposed by Fischler and Elschlager [23] for identifying structured objects in images. Felzenszwalb and Huttenlocher improved the matching efficiency of the parts based algorithm using distance transforms within dynamic programming [24]. An adaptation of the model is presented here and we apply it to find the bodyparts in pressure images.

A graph model can be used to represent the human body. Each part of the human body can be represented as a node in a graph, e.g., shoulders, hips, upper legs, and lower legs are nodes connected by edges. In this method, the appearance of a bodypart $j$ is measured using a mismatch cost $m_{j}\left(I, l_{j}\right)$ where $I$ is the image and $l_{j}=\left(x_{j}, y_{j}, \theta_{j}\right)$ is the center location and rotation of the bodypart. A low value of mismatch means the bodypart model is well recognized at that location and rotation. The calculation for mismatch cost is described in Section IV-A1.

In addition to this appearance cost for each bodypart model, there is a cost which measures the relationship between bodypart locations, called the deformation cost $d_{i j}\left(l_{i}, l_{j}\right)$. For bodyparts $i$ and $j$, it gives a measure of the error of their expected separation. For instance, the hip and shoulders are known to be separated by the spine length. The calculation for deformation cost is described in Section IV-A.

So for a given set of bodypart models and relationships between them, the best configuration of bodyparts $L=$ $\left(l_{1}, \ldots, l_{n}\right)$ in an image minimizes the total cost of mismatch for all parts and deformation between all pairs of parts

$$
\arg \min _{L}\left(\sum_{\text {allparts }} m_{j}\left(I, l_{j}\right)+\sum_{\text {allpairs }} d_{i j}\left(l_{i}, l_{j}\right)\right) .
$$

For $n$ bodyparts, the calculation of this expression grows exponentially. However, with the tree representation of bodyparts, it can be solved more efficiently as a chain of computations using dynamic programming. For any leaf node (having no children), its best location $\hat{l}_{j}$ can be calculated as a function of its parent location $l_{i}$. So the best leaf node location is a function of parent location

$$
B_{j}\left(l_{i}\right)=\arg \min _{l_{j}}\left(m_{j}\left(I, l_{j}\right)+d_{i j}\left(l_{i}, l_{j}\right)\right) .
$$

For a nonleaf, nonroot node, its best location $\hat{l}_{j}$ as a function of its parent location $l_{i}$ is given by

$$
B_{j}\left(l_{i}\right)=\arg \min _{l_{j}}\left(m_{j}\left(I, l_{j}\right)+d_{i j}\left(l_{i}, l_{j}\right)+\sum_{\mathrm{Ch}(\mathrm{j})} B_{c}\left(l_{j}\right)\right)
$$

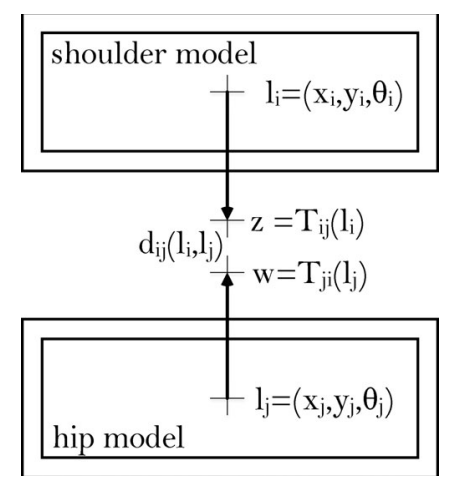

Fig. 3. Transformation of bodypart models. The upper box represents the shoulder region and the lower box represent the hip region. The locations of the bodypart models are transformed into a common space, which allows the calculation of a deformation cost.

where $\mathrm{Ch}(j)$ means children of $j$, and $B_{c}\left(l_{j}\right)$ are the costs of the best locations of the children of $j$. This is already calculated and memorized via the dynamic programming methodology.

Then, for the root node (no parent), its best location is

$$
\hat{l}_{j}=\arg \min _{l_{j}}\left(m_{j}\left(I, l_{j}\right)+\sum_{C h(j)} B_{c}\left(l_{j}\right)\right)
$$

where $B_{c}\left(l_{j}\right)$ is known for each of the children of $j$. This formulation, (2)-(4), reduces the number of computations from exponential to polynomial while still producing the globally best solution.

1) Compute Mismatch Cost: To compute the mismatch cost $m_{j}\left(I, l_{j}\right)$ for each bodypart, we select a representative template. The bodyparts are simply represented as rectangular boxes surrounded by a border (see Fig. 3 which shows an example of the shoulder and hip models). To account for different pressure images with varying weights of subjects, the image $I$ is binarized at different thresholds. A convolution operation of this kernel with the binary images efficiently gives the mismatch cost at all locations. This computes how many pixels do not match inside the inner box and within the border. Then, the mismatch cost is the smallest cost across all threshold levels for each location.

2) Compute Deformation Cost: To compute the deformation cost $d_{i j}\left(l_{i}, l_{j}\right)$ between bodyparts, we define this cost to have the form

$$
d_{i j}\left(l_{i}, l_{j}\right)=\left\|T_{i j}\left(l_{i}\right)-T_{j i}\left(l_{j}\right)\right\| .
$$

This is a simple distance metric between locations transformed to a common space (refer to Fig. 3). The transforms can be translations, rotations, and scaling, hence they are invertible. The effect of the transforms is to specify the expected relations between the locations, i.e., if two bodyparts have no deformation cost and are correctly positioned, then the transformed locations will coincide. The pairwise distance metric takes quadratic time in the number of locations. For every location of the parent, we want to compute the distance to each location of the child. On the surface, quadratic time may seem satisfactory but this can be improved to linear time through the use of distance transforms [25]. In fact, every pairwise distance between $l_{i}$ and $l_{j}$ need not 
be computed. From (2), only the smallest sum of deformation cost and mismatch cost is needed at each location of $l_{i}$.

The generalized form of a distance transform given a function $f$ is

$$
D(z)=\min _{w}(\|z-w\|+f(w))
$$

where $z$ and $w$ are locations in a grid. This formulation states that, for every location $z$, it finds the closest location $w$ with the smallest value for $f(w)$.

In (6), we can map $z$ to a translated location for $l_{i}$, i.e., $z=$ $T_{i j}\left(l_{i}\right)$. $w$ maps to a translated location for $l_{j}$, i.e., $w=T_{j i}\left(l_{j}\right)$. $f(w)$ is a function that maps to $f(w)=m_{j}\left(I, T_{j i}^{-1}\left(l_{j}\right)\right)$.

With these equivalences, we write (2) in a form where the generalized distance transform applies

$$
\begin{aligned}
B_{j}\left(l_{i}\right)=D\left(T_{i j}\left(l_{i}\right)\right)= & \min _{l_{j}}\left(\left\|T_{i j}\left(l_{i}\right)-T_{j i}\left(l_{j}\right)\right\|\right. \\
& \left.+m_{j}\left(I, T_{j i}^{-1}\left(T_{i j}\left(l_{i}\right)\right)\right)\right) .
\end{aligned}
$$

For nodes that are not leaves or root, (3) converts to

$$
\begin{aligned}
B_{j}\left(l_{i}\right)=D\left(T_{i j}\left(l_{i}\right)\right)= & \min _{l_{j}}\left(\left\|T_{i j}\left(l_{i}\right)-T_{j i}\left(l_{j}\right)\right\|\right. \\
& +m_{j}\left(I, T_{j i}^{-1}\left(T_{i j}\left(l_{i}\right)\right)\right) \\
& \left.+\sum_{C h(j)} B_{c}\left(T_{j i}^{-1}\left(T_{i j}\left(l_{i}\right)\right)\right)\right) .
\end{aligned}
$$

Now, the final discussion describes how to speed up the calculation of (7) and (8). For the leaf nodes, we initialize an array $D[x, y, \theta]$ with the values of $m_{j}\left(I, T_{j i}^{-1}\left(T_{j i}\left(l_{j}\right)\right)\right)$, which is just the mismatch cost of the child but translated to a new location in the same space as the parent. For nonleaf nodes, we initialize the array $D[x, y, \theta]$ with the values of

$$
m_{j}\left(I, T_{j i}^{-1}\left(T_{i j}\left(l_{i}\right)\right)\right)+\sum_{C h(j)} B_{c}\left(T_{j i}^{-1}\left(T_{i j}\left(l_{i}\right)\right)\right)
$$

which is the mismatch cost of the child (translated to a new location in the same space as the parent) plus the cost of the children. The values of $D[x, y, \theta]$ can be updated according to

$$
\begin{aligned}
D[x, y, \theta]= & \min (D[x, y, \theta], \\
& D[x-1, y, \theta]+k_{x}, \\
& D[x, y-1, \theta]+k_{y}, \\
& \left.D[x, y, \theta-1]+k_{\theta}\right)
\end{aligned}
$$

where $k_{x}, k_{y}, k_{\theta}$ are constants that account for different pixel scales. This is executed in sequence starting from array point $[1$, $1,1]$ linearly through the array, which is followed by a second sweep in the reverse direction.

With this computation, we have found the best location of the child for all locations of parents. Continuing this pattern for all vertices, the best location of the root can be solved efficiently, and the best location of the children are traced from the parents.

\section{B. Respiratory Signal Extraction}

This section describes the respiratory signal extraction method from the time series pressure regions. This results in a pressure indicator which is based on an observation that, while breathing, the pressure distribution along with vertical direction (from chest to stomach direction) varies with certain patterns and rhythms. For every frame, the pressure indicator sums the pressure values with their corresponding vertical directional coordinates

$$
\operatorname{Resp}(t)=\sum_{(x, y) \in \text { torso }} \sum_{* I[x, y](t) .}
$$

The range of the pixels in the torso is the region between chest and hip bounding boxes, which is enclosed by the bottom of the shoulder bounding box and the top of the hip bounding box. The calculated pressure indictor forms a time-series respiratory signal stream.

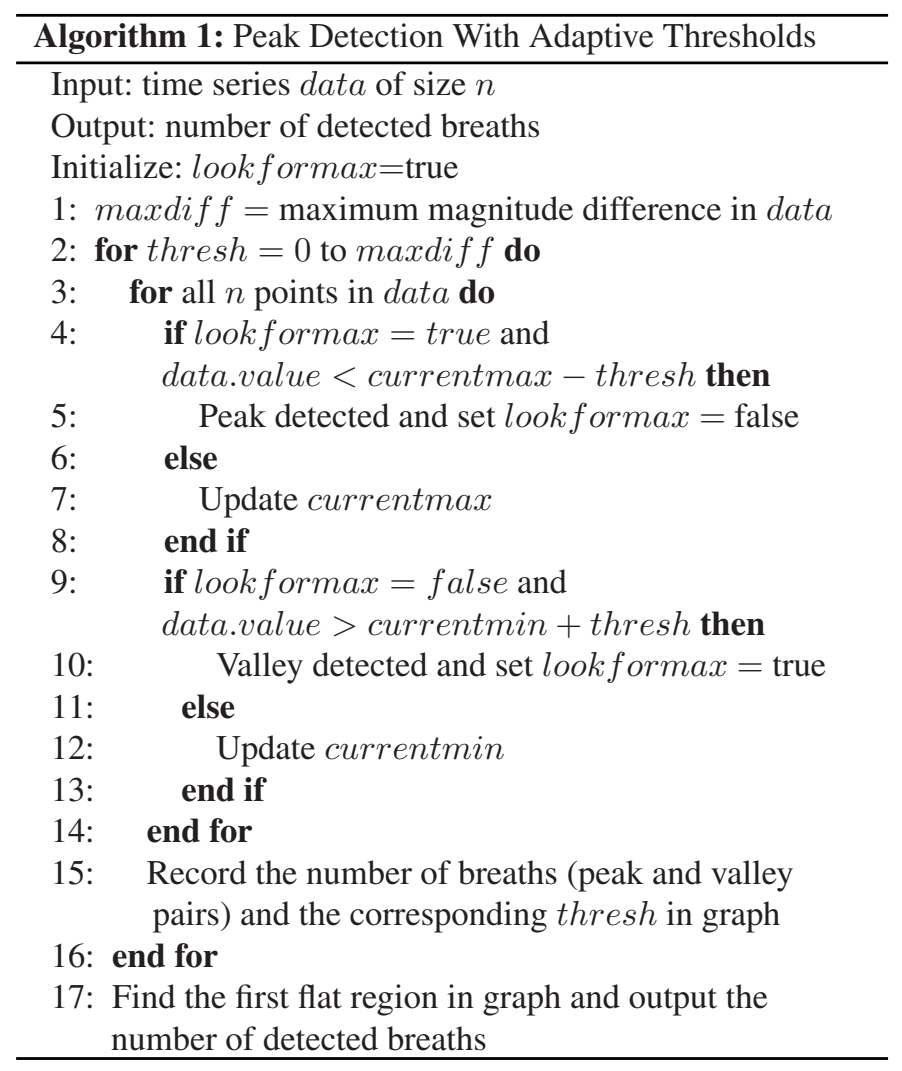

\section{Peak Detection With Adaptive Thresholding}

In general, a simple peak detection algorithm [26] is sufficient to recognize the periodic peak and valley of the time series data. However, arbitrary thresholding does not always work since the magnitude difference between peak and valley of time series data varies among testing subjects. Subjects breathing with moderate muscle activity in their shoulder or back area tend to generate large magnitude differences in the calculated pressure indictor time series. To accommodate the differences among subjects, the selected peak detection algorithm is augmented with adaptive threshold version as shown in the Algorithm 1.

Given a time series data of the extracted respiration signal, this adaptive threshold method profiles the relationship between the number of breaths and threshold values. A threshold is 


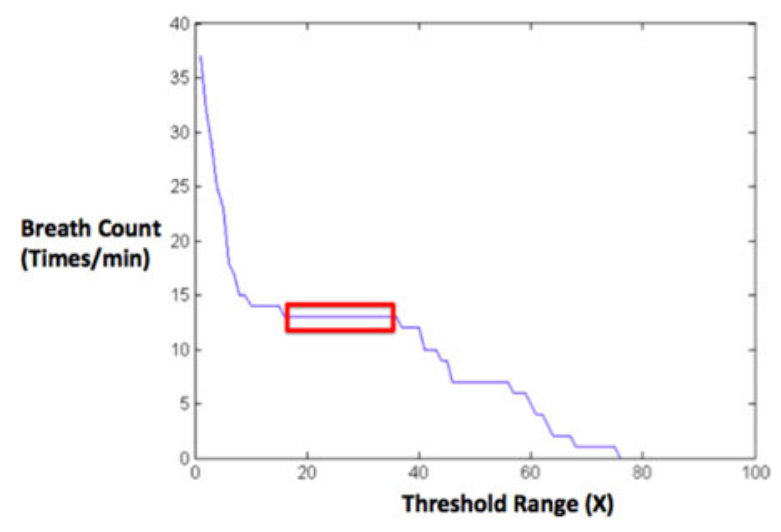

Fig. 4. Number of detected breaths for threshold values.

defined as the value difference by which a peak is recognized from surrounding data. So for each threshold value between 0 and the maximum magnitude difference in the data, the algorithm detects a peak when the data falls below the peak value by more than the threshold value. Similarly, a valley is detected when the data rises above the valley value by more than the threshold. Once all thresholds have been tested, it then finds the range of thresholds that have the least effect on the number of breathing events. This coincides with the flat area (see Fig. 4). It means that, within certain threshold ranges, the number of breathing events is consistent and implies those thresholds are less sensitive to the random hardware noise. The number of breathing events is recorded and the corresponding event intervals are used to calculate respiratory rate. A period of peak and valley is viewed as a complete respiration and the intervals between two peaks are viewed as breath intervals.

\section{EXPERIMENTS}

Twelve subjects participated in this study with heights 152 $190 \mathrm{~cm}$, weights 46-83 kg. The high-density pressure sensor sheet was deployed on top of a foam mattress with memory in a lab environment. The experimental goals were to evaluate the effectiveness of the torso localization algorithm for respiratory signals extraction on a regular soft bed environment. During experiments, subjects were allowed to lie in their preferred posture and keep their regular lying habits. In addition, data were recorded in full without segmentation and truncation due to interferences caused by extremity movements. Full experiment processes were taped, breath events were timed and labelled manually from the taped videos according to the visible chest wall movement. Recording started when the subjects lied on top of the pressure sensing system in their preferred lying posture. They were asked to breathe as usual and not to make any large posture changes, such as rolling or sliding, during the recording. Furthermore, tilted bed situations as commonly seen in clinics were simulated by changing the tilted angles of the bed head and foot areas. For these tilted bed situations, the subjects were evaluated in supine posture only.
TABLE I

RESPIRATION RESUlTS In THREE COMMON Lying POSTURES (Detected Breaths/Ground TRUth) Over 10-Min Periods

\begin{tabular}{lccc}
\hline \hline ID & Supine & Lateral & Prone \\
\hline 1 & $174 / 176$ & $169 / 172$ & $176 / 177$ \\
2 & $147 / 155$ & $137 / 154$ & $143 / 152$ \\
3 & $171 / 175$ & $162 / 173$ & $170 / 175$ \\
4 & $184 / 185$ & $184 / 186$ & $187 / 188$ \\
5 & $127 / 130$ & $125 / 133$ & $130 / 127$ \\
6 & $136 / 142$ & $138 / 144$ & $139 / 144$ \\
7 & $155 / 162$ & $162 / 162$ & $157 / 160$ \\
8 & $179 / 177$ & $174 / 176$ & $172 / 175$ \\
9 & $160 / 166$ & $158 / 167$ & $162 / 166$ \\
10 & $138 / 144$ & $137 / 142$ & $139 / 141$ \\
11 & $164 / 168$ & $170 / 172$ & $168 / 172$ \\
12 & $152 / 150$ & $147 / 146$ & $148 / 148$ \\
Avg. & $\mathbf{1 5 7 . 5 / 1 6 0 . 8}$ & $\mathbf{1 5 5 . 3 / 1 6 0 . 6}$ & $\mathbf{1 5 7 . 6 / 1 6 0 . 4}$ \\
Error & $\mathbf{2 . 0 \%}$ & $\mathbf{3 . 3 \%}$ & $\mathbf{1 . 8 \%}$ \\
\hline \hline
\end{tabular}

\section{A. Results for Respiration Monitoring}

Pressure values variation within the torso region is of most interest for respiratory signal extraction. In this experiment, respiratory signals were extracted from pressure image sequences when participants lied in common lying postures: supine, side, and prone for $10 \mathrm{~min}$ each. The method of torso localization was applied to the pressure image sequences to keep track of the regions between chest and stomach areas. Then, signal extraction via vertical weighting calculation and peak detection methods were used to extract the breathing events. Table I summarizes the results and shows comparisons between the detected respiration events and the ground truth visually recognized from video recordings for all three lying postures. On average, the detected respiration events match well with ground truth across the three common lying postures. Different lying postures and extremities movements did not affect the quality of torso localization and the extracted respiratory signals.

\section{B. Visualization of Respiration Motion}

It is useful to illustrate the effect that vertical weighting calculation makes on respiratory signal extraction. There is an apparent geometrical delay from chest to stomach area and weighted pressure values with its vertical coordinates relative to the bounding box border emphasizes the respiration pattern. Visualizing the vertical momentum calculation can be a useful tool to investigate the nature of the geometrical delay phenomenon in pressure image sequences.

In order to visualize calculated vertical momentum, results from peak detection algorithm are used. Peak detection algorithm returns a series of peaks and valleys from the pressure indicator time series. By subtracting two consecutive peak and valley indexed pressure images, the differences between images of peaks and valleys are obtained. Fig. 5 visualizes the peaks and valleys of respiration by averaging the pressure differences between peaks and valleys. To better visualize the difference of chest and stomach area, all positive differences were marked as black and negative differences were marked as white. Gray area stands for no obvious pressure differences. It can be seen 


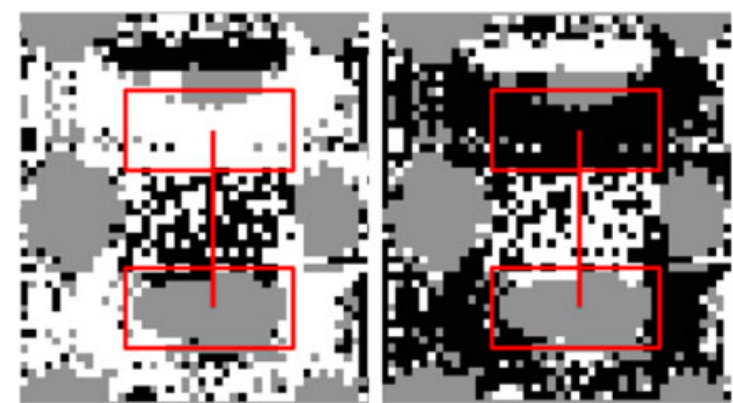

Fig. 5. Examples of change in pressure distribution for inhaling and exhaling events. The red boxes show the location of detected shoulder and hip regions. The black and white areas show the locations of greatest pressure change.

TABLE II

ReSUlts SHOW HOW THE REGION OF EXTRACTED DATA AFFECTS THE QUALITY OF BREATH COUNTS

\begin{tabular}{lccc}
\hline \hline ID & Fullsheet & Center of Mass & Torso Area \\
\hline 1 & 11 & 10 & 2 \\
2 & 14 & 9 & 4 \\
3 & -8 & -6 & -3 \\
4 & -8 & -4 & -1 \\
5 & 12 & 8 & 3 \\
6 & -7 & -2 & -2 \\
7 & 6 & 1 & 3 \\
8 & -6 & -5 & -3 \\
9 & 11 & 9 & 4 \\
10 & 8 & 8 & 2 \\
11 & 4 & 5 & 3 \\
12 & -9 & -7 & -2 \\
Ave. & $\mathbf{9 . 1 8}$ & $\mathbf{6 . 6 7}$ & $\mathbf{2 . 7 9}$ \\
Error & $\mathbf{5 . 7 \%}$ & $\mathbf{4 . 1 \%}$ & $\mathbf{1 . 7 \%}$ \\
\hline \hline
\end{tabular}

This table shows the difference in counts between estimated breathing and the ground truth over 10-min periods. The recordings contain $160-180$ breaths. Three regions of feature extraction are tested and the torso region shows the least error

that pressure distribution of chest area is in opposite phase of the stomach area. This opposite phase phenomenon explains the reason why vertical weighting calculations can be a useful indicator for respiratory patterns extraction, because the geometrical delay from chest to stomach region is included in the indicator calculation.

\section{Comparison of Body Region Locations}

Three scenarios were evaluated and compared to demonstrate the importance of torso tracking: signals from the whole sheet without applying torso localization algorithm; signals from half of the sheet and centralized in the center of the weight; and signals from torso localized area, same as the results presented previously. The difference between the estimated breathing events and ground truth are reported in Table II for the supine posture.

Extracted respiratory events from torso localized areas showed good consistency with the ground truth. This is because during breathing, large periodic pressure variances are expected in the chest to stomach regions. Pressure variance outsides the torso regions can be viewed as interference, which directly im-

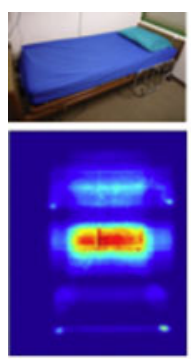

(a)

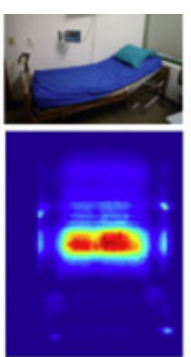

(b)

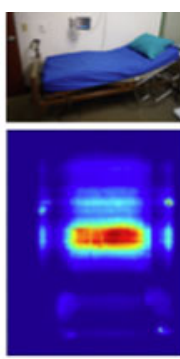

(c)

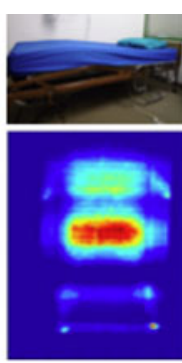

(d)
Fig. 6. Tilted bed setup. (a) Original. (b) HeadLift. (c) EndLift. (d) BothLift.

pacts the calculation of vertical weighted signal extraction. Interference caused by the extremities movements were time-varied and subject-dependent; therefore, the accuracy of respiratory signal extraction from the whole sheet largely was highly impacted by the movements of the participants. For the case of extracting signals from the area nearby the center of mass, the accuracy of respiratory signal extraction was reduced because the targeted area included parts of extremities. In summary, respiratory signal extraction with torso localization effectively reduces the interference caused by the extremities movement, and the accuracy of respiratory signals were less varied across subjects.

\section{Respiration in Tilted Bed Environments}

Another common scenario of sleep environment in clinics is tilted bed setup. Tilted bed environments change the original pressure distribution underneath a human body. Hence, it is necessary to evaluate the effect of tilted environment on respiratory signal extraction. Fig. 6 shows pressure image samples from tilted environment with raised head $\left(15^{\circ}\right)$, raise knee $\left(15^{\circ}\right)$, and both raised head and knee. Although pressure distribution changed with degree of tilting, the areas of chest and stomach were still visually recognizable. Pressure redistribution caused by tilted bed environment has limited effect on body contact area size and the pressure values in chest and hips areas are still prominent compared to other regions. However, the major difference is the distribution of pressure within the body profile.

In this experiment, participants were requested to lie in supine position for $5 \mathrm{~min}$ of recording. Results of Fig. 7 reveal that respiratory signals can be extracted from tilted bed environment and show consistency. Actually, it is worth noticing that much under body pressure was accumulated in the chest area for the case of raised knee tilting; hence, the interference caused by the upper extremities movements had larger weight in the vertical weighting calculation. On the other hand, much under body pressure was accumulated in the stomach and hip area for the case of the raised head tilting; hence, the interference caused by the lower extremities movements had larger impact on the respiratory signals extraction. Therefore, torso localization becomes more important in a tilted bed environment because redistributed pressure tends to increase the weight of extremity movements. Slight extremities movement may incur large interference to respiratory signal extraction. 


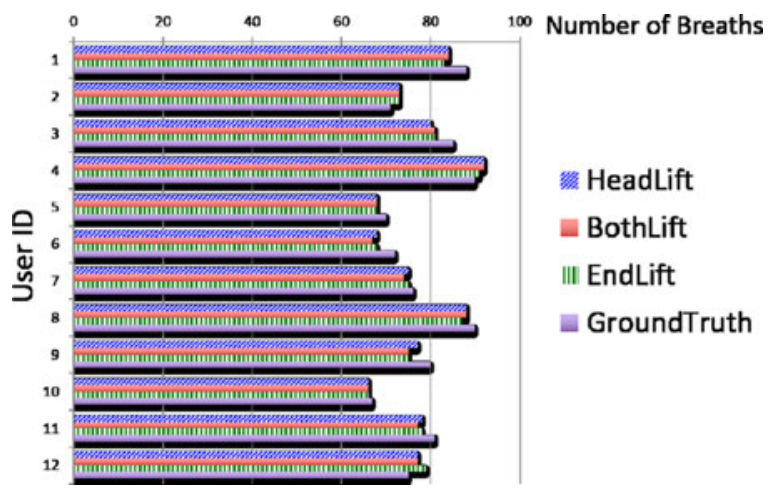

Fig. 7. Breath count comparison for each subject on tilted beds.

\section{CONCLUSION AND Future WorK}

In this paper, we present BreathSens, a continuous and unobtrusive system that monitors on-bed respiration using a highdensity pressure bedsheet made of e-Textile. Our experimental results show that breathing events are accurately identified in several different situations. All sleep movements are recorded as pressure images detected by the e-Textile bed sheet and are analyzed via pressure images analysis, namely torso localization, respiration signal extraction, and peak detection. The complete system design can promote clinical or at-home sleep condition monitoring and assist in notifications due to possible breathingrelated disorders. It could be used for situations, such as sleep apnea screening to alert for possible medical diagnoses.

Tracking a moving subject is difficult in the current stage, because body movement largely redistributes the pressure between the human body and the sensing system. For some applications, such as sleep apnea screening, losing respiration tracking for a moving subject is acceptable. However, monitoring respiration during movement can be an important future work. Although high-density pressure sensors are required for accurate localization, other future work includes optimizing the number of sensors while providing an adequate level of performance.

\section{ACKNOWLEDGMENT}

The authors would like to thank Medisens Wireless Inc. for their contributions in discussion, building, and supply of the hardware.

\section{REFERENCES}

[1] M. Peberdy, W. Kaye, J. Ornato, G. Larkin, V. Nadkarni, M. Mancini, R. Berg, G. Nichol, and T. Lane-Trultt, "Cardiopulmonary resuscitation of adults in the hospital: A report of 14720 cardiac arrests from the national registry of cardiopulmonary resuscitation," Resuscitation, vol. 58, no. 3, pp. 297-308, 2003.

[2] K. Reed, and R. May, "Healthgrades seventh annual patient safety in American hospitals study," HealthGrades, Inc., Denver, CO, US, 2011.

[3] T. J. Hodgetts, G. Kenward, I. G. Vlachonikolis, S. Payne, and N. Castle, "The identification of risk factors for cardiac arrest and formulation of activation criteria to alert a medical emergency team," Resuscitation, vol. 54, no. 2, pp. 125-131, 2002.

[4] J. Fieselmann, M. Hendryx, C. Helms, and D. Wakefield, "Respiratory rate predicts cardiopulmonary arrest for internal medicine patients," J. Gen. Internal Med., vol. 8, no. 7, pp. 354-360, 1993.
[5] M. Cretikos, J. Chen, K. Hillman, R. Bellomo, S. Finfer, and A. Flabouris, "The objective medical emergency team activation criteria: A case-control study," Resuscitation, vol. 73, no. 1, pp. 62-72, 2007.

[6] M. Cretikos, R. Bellomo, K. Hillman, J. Chen, S. Finfer, and A. Flabouris, "Respiratory rate: The neglected vital sign," Med. J. Aust., vol. 188, no. 11, pp. 657-659, 2008.

[7] J. McBride, D. Knight, J. Piper, and G. B. Smith. (2005). Long-term effect of introducing an early warning score on respiratory rate charting on general wards. Resuscitation. [Online]. 65(1), pp. 41-44. Available: http://www.sciencedirect.com/science/article/pii/S0300957204004381

[8] F. AL-Khalidi, R. Saatchi, D. Burke, H. Elphick, and S. Tan, "Respiration rate monitoring methods: A review," Pediatr. Pulmonol., vol. 46, no. 6 , pp. 523-529, 2011.

[9] Covidien. (2012). Nellcor bedside respiratory patient monitoring system. [Online]. Available: http://www.covidien.com

[10] S. DeMeulenaere, "Pulse oximetry: Uses and limitations," J. Nurse Pract., vol. 3, no. 5, pp. 312-7, 2007.

[11] J. S. Cardozo, "New AASM recommendations for sensors: A simple guide for the sleep technologist," Sleep Diagnosis Therapy, vol. 21, no. 3, pp. $21-22,2008$.

[12] Y. Chee, J. Han, J. Youn, and K. Park, "Air mattress sensor system with balancing tube for unconstrained measurement of respiration and heart beat movements," Physiol. Meas., vol. 26, no. 4, pp. 413-422, 2005.

[13] A. Hart, K. Tallevi, D. Wickland, R. Kearney, and J. Cafazzo, "A contactfree respiration monitor for smart bed and ambulatory monitoring applications," in Proc. IEEE Annu. Int. Conf. Eng. Med. Biol. Soc., 2010, pp. 927-930.

[14] M. Nishyama, M. Miyamoto, and K. Watanabe. (2011). Respiration and body movement analysis during sleep in bed using heterocore fiber optic pressure sensors without constraint to human activity. J. Biomed. Opt. [Online]. 16(1), pp. 17002-17007. Available: http://dx.doi.org/10.1117/1.3528008

[15] W. Xu, C. Gu, C. Li, and M. Sarrafzadeh, "Robust doppler radar demodulation via compressed sensing," Electron. Lett., vol. 48, no. 22, pp. 1428-1430, 2012.

[16] M.-Z. Poh, D. J. McDuff, and R. W. Picard, "Non-contact, automated cardiac pulse measurements using video imaging and blind source separation," Opt. Exp., vol. 18, pp. 10762-10774, 2010.

[17] Philips. (2013). Vital signs camera. [Online]. Available: http://www. vitalsignscamera.com

[18] R. Murthy, I. Pavlidis, and P. Tsiamyrtzis, "Touchless monitoring of breathing function," in Proc. IEEE Annu. Int. Conf. Eng. Med. Biol. Soc., 2004, pp. 1196-1199.

[19] M. Rofouei, W. Xu, and M. Sarrafzadeh, "Computing with uncertainty in a smart textile surface for object recognition," in Proc. IEEE Conf. Multisens. Fusion Integr. Intell. Syst., Sep. 2010, pp. 174-179.

[20] M. Huang, J. Liu, W. Xu, N. Alshurafa, X. Zhang, and M. Sarrafzadeh, "Using pressure map sequences for recognition of on bed rehabilitation exercises," IEEE J. Biomed. Health Informat., vol. 18, no. 2, pp. 411-418, Mar. 2014.

[21] W. Xu, Z. Li, M. Huang, N. Amini, and M. Sarrafzadeh, "Ecushion: An etextil device for sitting posture monitoring," in Proc. Int. Conf. Body Sens. Netw., 2011, pp. 194-199.

[22] J. Liu, W. Xu, M. Huang, N. Alshurafa, and M. Sarrafzadeh, "A dense pressure sensitive bedsheet design for unobtrusive sleep posture monitoring," in Proc. IEEE Int. Conf. Pervasive Comput. Commun., 2013, pp. 207-215.

[23] M. A. Fischler and R. Elschlager, "The representation and matching of pictorial structures," IEEE Trans. Comput., vol. C-22, no. 1, pp. 67-92, 1973.

[24] P. F. Felzenszwalb and D. P. Huttenlocher, "Pictorial structures for object recognition," Int. J. Comput. Vision, vol. 61, pp. 55-79, 2005.

[25] G. Borgefors, "Distance transformations in digital images," Comput. Vis., Graph. Image Process., vol. 34, no. 3, pp. 344-371, 1986.

[26] E. Billauer. (2008). peakdet: Peak detection using MATLAB. [Online]. Available: http://www.billauer.co.il/peakdet.html.

Authors' photographs and biographies not available at the time of publication. 